\title{
The Evaluation of Magee Equation 2 in Predicting Response and Outcome in Hormone Receptor-Positive and HER2-Negative Breast Cancer Patients Receiving Neoadjuvant Chemotherapy
}

This article was published in the following Dove Press journal: Cancer Management and Research

\author{
Napat Saigosoom ${ }^{1, *}$ \\ Doonyapat Sa-nguanraksa ${ }^{1, *}$ \\ Eng O-charoenrat ${ }^{2}$ \\ Thanawat Thumrongtaradol ${ }^{1}$ \\ Pornchai O-charoenrat (iD) \\ 'Division of Head, Neck and Breast \\ Surgery, Department of Surgery, Faculty \\ of Medicine, Siriraj Hospital, Mahidol \\ University, Bangkok, 10700, Thailand; \\ ${ }^{2}$ Faculty of Medical Sciences, University \\ College London, London WCIE 6BT, UK \\ *These authors contributed equally to \\ this work
}

Background and Purpose: Magee Equations have been developed as accurate tools for predicting response and clinical outcomes in breast cancer patients treated with adjuvant systemic therapy using basic clinicopathological parameters. This study aims to evaluate the alternative application of Magee Equation 2 score in predicting pathological complete response (pCR) after neoadjuvant chemotherapy (NAC) in hormone receptor (HR)-positive, HER2negative breast cancer.

Patients and Methods: Patients with HR-positive, HER2-negative breast cancer who received NAC from January 2010 to May 2018 at Siriraj Hospital, Mahidol University, Thailand, were recruited. Pre-treatment status of HR and HER2 was used to calculate the Magee Equation 2 scores. The pCR rates among different clinicopathological parameters were analyzed. Survival analysis was performed by Log-rank test. Kaplan-Meier survival curves were analyzed.

Results: A total of 215 patients were eligible. The pCR rates for low, intermediate, and high scores were $4.8 \%, 3.6 \%$, and $23.8 \%$, respectively. Patients with high scores had significantly higher size reduction and pCR rates compared to those with intermediate or low scores $(p<0.001)$. Those with high scores had higher rates of locoregional recurrence and death. The patients with high score had significantly lower overall survival $(p=0.034)$.

Conclusion: Among patients with HR-positive and HER2-negative breast cancer treated with NAC, Magee Equation 2 might be used as a tool for predicting the pCR and clinical outcome.

Keywords: breast cancer, Magee Equation, neoadjuvant chemotherapy, pathologic complete response, survival

\section{Introduction}

Breast cancer is the most common cancer in women worldwide and it is the leading cause of cancer-related death among females worldwide. ${ }^{1}$ Estrogen receptor (ER), progesterone receptor, and HER2 are considered as important biomarkers that can predict not only the response to treatment but also prognosis and disease recurrence. ${ }^{2-4}$ $\mathrm{Ki}-67$ is known to be present in all proliferating cells and it has been used as a surrogate marker to assess proliferation index. 5,6

Approximately $75 \%$ of invasive breast cancers are ER-positive and/or PR-positive tumors. ${ }^{7}$ Although approximately $85 \%$ of these women may be recurrence free at
Correspondence: Pornchai O-charoenrat Division of Head Neck and Breast Surgery, Department of Surgery, Faculty of Medicine, Siriraj Hospital, Mahidol University, 2 Wanglang Road, Bangkoknoi, Bangkok 10700, Thailand

Tel +6624192837

Email pornchai.och@mahidol.ac.th 
10 years with adjuvant hormonal therapy alone, the addition of chemotherapy leads to relative reduction in the risk of recurrence of approximately $30 \%$ on average. This leads to an absolute benefit for an individual patient ranging from $1 \%$ to $5 \% .{ }^{8,9}$ Many patients with ER-positive breast cancer would, therefore, be over treated with chemotherapy based on clinicopathologic features alone although most of them would have been adequately treated with hormonal therapy alone. ${ }^{10,11}$ On the other hand, neoadjuvant chemotherapy (NAC) before surgery is the standard therapy in the patients with locally advanced breast cancer ${ }^{12}$ and also increased the rate of breast conserving surgery in early breast cancer. ${ }^{13}$ The patients with pathological complete response (pCR) after NAC have improved survival. ${ }^{14}$

Gene expression profile assessed by the 21-gene assay (Oncotype Dx ${ }^{\circledR}$; Genomic Health, Redwood City, CA) has been developed to precisely identify patients with ER-positive breast cancer who will benefit from chemotherapy using the result expressed as a recurrence score (RS). ${ }^{15}$ Furthermore, $R S$ can discriminate pathological complete response $(\mathrm{pCR})$ in the patients who received NAC. ${ }^{16}$ However, it has limitations, including a cost of over $\$ 4000$ per test and a delay in treatment while waiting for the results. A recent study reported that biomarkers including ER, PR, and HER-2 can be incorporated into a multivariable model (known as Magee Equations; http:// path.upmc.edu/onlineTools/MageeEquations.html) to predict the RS. ${ }^{17,18}$ The Magee Equations have been studied in both internal and external validation studies. ${ }^{19,20}$ Since the 21 -gene assay has been shown to predict the benefit of adjuvant chemotherapy in women with hormone-receptor (HR)-positive, HER2-negative, axillary node-negative breast cancer, we hypothesized that Magee Equation 2 should also predict the response to NAC. This study aims to evaluate the alternative application of Magee Equation 2 score in predicting pCR after NAC in HR-positive, HER2-negative breast cancer.

\section{Patients and Methods}

\section{Patients}

This study was approved by Siriraj Institutional Review Board. The patient consent to review their medical records was not required due to this study involved the collection of existing data recorded by the investigator in such a manner that subjects cannot be identified, directly or through identifiers linked to the subject. This study was conducted in accordance with the Declaration of Helsinki. Patients with HR-positive, HER2-negative breast cancer who received NAC from January 2010 to May 2018 at Siriraj Hospital,
Mahidol University, Thailand were retrospectively reviewed. pCR was defined as the absence of invasive tumor in the surgical specimen (pCR in both breast and axillary nodes). Residual carcinoma in situ without invasive carcinoma was allowed for $\mathrm{pCR}^{21}$ Estimated tumor size reduction in the breast was calculated using the following equation: Estimated percent tumor size reduction $=(($ pre-therapy clinical size-pathology size)/pre-therapy clinical size) x100.

The pathology size is the largest dimension of the gross tumor bed by the invasive tumor cellularity. Pre-treatment status of ER, PR, HER2, and Ki-67 was available from core biopsy pathology reports and were used to calculate Magee Equation 2 scores using the equation:

Magee Equation 2 score $=18.8042+$ Nottingham score $\mathrm{x} 2.34123$

$+\mathrm{ERH}-\operatorname{score} \mathrm{x}(-0.03749)+\mathrm{PR} \mathrm{H}-\operatorname{score} \mathrm{x}(-0.03065)$

$+(0$ for HER $2-$ negative, 1.82921 forequivocal, 11.51378 for

HER 2 - positive) + tumor size $x 0.04267$

The H-score was calculated by summation of the results of multiplication of the percentage of cells $(0-100 \%)$ with staining intensity ( 0 for negative, 1 for weak, 2 for moderate, and 3 for strong intensity). The results can be ranged from 0 to $300 .{ }^{22}$ Magee Equation 2 scores were divided into 3 categories according to the $R S$ obtained from Oncotype Dx: $0-<18=$ low; $18-<31=$ intermediate; and $\geq 31=$ high .

\section{Statistical Analysis}

Statistical analysis was performed using SPSS version $25.0^{\circledR}$ (SPSS Inc., Chicago, USA). Continuous parameters were compared by independent $t$-test or Wilcoxon rank sum test. Categorical parameters were analyzed by chi-square statistics and the $p$-values were obtained from a two-sided Fisher exact test. Patients with missing or unknown information were excluded from the test. Confidence intervals were obtained using Wald normal approximation. Multivariable analysis for prediction of $\mathrm{pCR}$ was performed after including variables that were significant on univariable analysis. Survival analyses were performed using the Log-rank (univariate analysis) and Cox regression (multivariate analysis) methods. Two-sided tests were used in all analyses. The significance level was set at $P<0.05$.

\section{Results}

Breast cancer cohort was searched for the term NAC and resulted in 757 patients. Of the 757 patients, 215 patients with ER-positive, HER2-negative or equivocal were eligible for participation (excluding 75 patients without NAC; 293 patients with HR-negative or HER2-positive; 127 patients 
with de novo metastatic breast cancer; and 47 patients with incomplete data or incomplete treatment). The demographic and tumor characteristics data with respect to pCR for the 215 ER-positive, HER2-negative/equivocal patients are shown in Table 1.

NAC regimens were chosen at the discretion of the medical oncologists and resulting in different NAC regimens. However, the majority $(96.3 \%)$ received an anthracycline (AC) and a sequential anthracycline and taxane (AC-T) regimens. Five patients $(2.3 \%)$ had received neoadjuvant endocrine therapy for 6 months before surgery. After neoadjuvant treatment, 49 patients underwent breast conserving surgery, while 166 patients underwent total mastectomy. Axillary lymph node dissection was performed in 181 patients and sentinel lymph node biopsy was performed in 34 patients. All pathological reports of both breast conserving surgery and mastectomy specimens showed free surgical margin.

Overall, 17 patients (7.9\%) had pCR. The patients with pCR had significantly lower ER H-score and PR H-score ( $p=0.003$ and $p=0.027$, respectively). Of the 215 patients, the distribution of Magee Equation 2 scores was low in 62 $(28.8 \%)$, intermediate in $111(51.6 \%)$, and high in 42 (19.5\%) patients. The pCR rates for low, intermediate, and high Magee Equation 2 scores were 4.8\%, 3.6\%, and 23.8\%, respectively (Table 2). Patients with high Magee Equation 2 scores were more likely to have pCR compare to those with intermediate or low scores $(\mathrm{OR}=7.41,95 \%$ CI 2.63-20.91, $p<0.001)$. Subgroup analysis of those who received AC-based regimen showed that the patients with high scores were more likely to have pCR $(\mathrm{OR}=14.17,95 \% \mathrm{CI}$ $2.58-77.87, p=0.002)$. The rate of $\mathrm{pCR}$ was significantly higher in those who received AC-T chemotherapy than those who received AC-based chemotherapy (13.3\% vs $4.8 \%, p=0.031$, respectively). The estimated tumor size reduction was also significantly higher in the patients with high Magee Equation 2 score when compare to those with low and intermediate scores. Multivariate analysis by binary logistic regression showed that Magee Equation 2 score was the only independent predictor for pCR (Table 3). The factors that were utilized to calculate Magee 2 score (tumor size, Nottingham score, ER H-score, PR H-score, and HER2 status) were not included in this multivariate analysis.

Median follow-up time was 33 months (1-106 months). There were 30 locoregional recurrences, 59 distant metastasis, and 36 deaths occurred. Those with high Magee Equation 2 scores had higher rates of locoregional recurrence and death when compared to those with low/intermediate score
$(\mathrm{OR}=2.47,95 \%$ CI 1.05-5.78, $p=0.033$, and $\mathrm{OR}=2.57,95 \%$ CI 1.16-5.71, $p=0.018$, respectively) (Table 2). Five-year disease-free survival (DFS) rates for low, intermediate, and high Magee Equation 2 scores were 69.7\%, 50.8\%, and $54.9 \%$, respectively. The survival analysis by Log-rank test showed worse DFS for high Magee Equation 2 category but the level of statistically significant was not reached $(p=0.101)$. Five-year overall survival (OS) rates for low, intermediate, and high Magee Equation 2 scores were $80.2 \%, 81.0 \%$, and $61.8 \%$, respectively. Univariate analysis by Log-rank test showed that the patients with high Magee Equation 2 scores had significantly lower OS ( $p=0.034)$. The survival curves estimated by Kaplan-Meier method are shown in Figures 1 and 2. Multivariate analysis by Cox regression revealed that $\mathrm{N}$ stage and Magee Equation 2 score were the independent predictors for both DFS and OS. The factors that were utilized to calculate Magee 2 score (tumor size, Nottingham score, ER H-score, PR H-score, and HER2 status) were not included in this multivariate analysis (Tables 4 and 5).

\section{Discussion}

Achievement of pCR following NAC was associated with better DFS and OS, especially in the patients with triplenegative or HER2-positive breast cancer. ${ }^{23}$ Several clinicopathological parameters were reported to be the predictors for response to NAC. ${ }^{24,25}$ Luminal breast cancer subtype had lower response to NAC when compare to other subtypes. ${ }^{26,27}$

Although there were higher proportion of $\mathrm{T} 4$ breast cancer recruited in this current study, the rate of $\mathrm{pCR}$ in the current study was similar to the recent report of clinically node-positive breast cancer receiving NAC. ${ }^{25}$ However, approximately $70 \%$ of the patients received neoadjuvant $\mathrm{AC}$ regimen which resulted in lower response when compare to the addition of taxane-based regimen. ${ }^{28}$

Gene expression profiles assessed by Oncotype DX in the patients with locally advanced breast cancer who received neoadjuvant paclitaxel and doxorubicin revealed that high $R S$ were strongly associated with $\mathrm{pCR} .{ }^{16}$ In a study by Yardley et al, 108 patients with HER2-negative breast cancer who received neoadjuvant ixabepilone and cyclophosphamide were assessed for $\mathrm{pCR}$ rate. The patients with a high-risk score had a pCR rate of $26 \%$ compared to $0 \%$ in patients with low or intermediate risk scores. ${ }^{29}$ These patients were most likely to receive the greatest clinical benefit from NAC. However, the Oncotype DX carries a high cost and may not be widely available in Thailand. Utilization of conventional clinicopathological parameters derived from pretreatment 
Table I Patient and Tumor Characteristics

\begin{tabular}{|c|c|c|c|c|}
\hline & Total $n=2 \mid 5$ & No pCR $n=198 ; 92.1 \%$ & PCR $n=17 ; 7.9 \%$ & p value \\
\hline \multicolumn{5}{|l|}{ Continuous Variables: Mean (Std. Dev) } \\
\hline Age & $50.6(10.8)$ & $50.4(10.58)$ & $52.71(13.09)$ & 0.398 \\
\hline Pre-therapy tumor size & $44.7(28.1)$ & $44.42(26.35)$ & $48.52(45.30)$ & 0.623 \\
\hline ER H-score & $193.9(100.2)$ & $201.24(94.6)$ & $109.29(\mid 25.51)$ & 0.003 \\
\hline PR H-score & $125.5(102.1)$ & $130.22(120.2)$ & $71.35(84.65)$ & 0.027 \\
\hline Ki-67 labeling index in \% & $42(21.0)$ & $41.98(21.29)$ & $42.10(20.02)$ & 0.987 \\
\hline \multicolumn{5}{|l|}{ Categorical Variables: N (\%) } \\
\hline \multicolumn{5}{|l|}{ Menopausal Status } \\
\hline Premenopausal & $119(55.3)$ & III (56.I) & $8(47.1)$ & 0.474 \\
\hline Postmenopausal & $96(44.7)$ & $87(43.9)$ & $9(52.9)$ & \\
\hline \multicolumn{5}{|l|}{ Pre-Therapy T Stage } \\
\hline TI & $13(6)$ & $9(4.5)$ & $4(23.5)$ & 0.969 \\
\hline $\mathrm{T} 2$ & $82(38.1)$ & $78(39.4)$ & $4(23.5)$ & \\
\hline T3 & $26(12.1)$ & $25(12.6)$ & I (5.9) & \\
\hline $\mathrm{T} 4 \mathrm{a}$ & I $(0.5)$ & $\mathrm{I}(0.5)$ & 0 & \\
\hline $\mathrm{T} 4 \mathrm{~b}$ & $69(32.1)$ & $64(32.3)$ & $5(29.4)$ & \\
\hline $\mathrm{T} 4 \mathrm{c}$ & $4(1.9)$ & $4(2.0)$ & 0 & \\
\hline $\mathrm{T} 4 \mathrm{~d}$ & $20(9.3)$ & $17(8.6)$ & $3(17.6)$ & \\
\hline \multicolumn{5}{|l|}{ Pre-Therapy N Stage } \\
\hline No & $55(25.6)$ & $52(26.3)$ & $3(17.6)$ & 0.550 \\
\hline NI & $100(46.5)$ & $91(46)$ & $9(52.9)$ & \\
\hline N2 & $41(19.1)$ & $38(19.2)$ & $3(17.6)$ & \\
\hline N3 & $19(8.8)$ & $17(8.6)$ & $2(11.8)$ & \\
\hline \multicolumn{5}{|l|}{ Pre-Therapy Staging } \\
\hline I & $4(1.9)$ & $3(1.5)$ & I (5.9) & 0.360 \\
\hline ॥ & $82(38.1)$ & 77 (38.9) & $5(29.4)$ & \\
\hline III & $129(60.0)$ & $118(59.6)$ & II (64.7) & \\
\hline \multicolumn{5}{|l|}{ Pre-Therapy Nuclear Grade } \\
\hline Grade I & $16(7.4)$ & $14(7.1)$ & $2(11.8)$ & 0.985 \\
\hline Grade 2 & $127(59.1)$ & $118(59.6)$ & $9(52.9)$ & \\
\hline Grade 3 & $70(32.6)$ & $6(32.3)$ & $6(35.3)$ & \\
\hline Unknown & $2(0.9)$ & $2(1)$ & $2(0.9)$ & \\
\hline \multicolumn{5}{|l|}{ Histology } \\
\hline Ductal & $207(96.3)$ & $190(96)$ & $17(100)$ & 0.420 \\
\hline Lobular & $3(1.4)$ & $3(1.5)$ & 0 & \\
\hline Others & $5(2.3)$ & $5(2.5)$ & 0 & \\
\hline \multicolumn{5}{|l|}{ HER2 Status } \\
\hline Negative & I7I (79.5) & $157(79.3)$ & $14(82.4)$ & 0.697 \\
\hline Equivocal $^{\mathrm{a}}$ & $44(20.5)$ & $4 \mid(20.7)$ & $3(17.6)$ & \\
\hline \multicolumn{5}{|l|}{ Neoadjuvant Chemotherapy Regimen } \\
\hline AC-based regimen & $147(68.4)$ & $140(70.7)$ & $7(4 \mid .2)$ & 0.044 \\
\hline AC-T regimen & $60(27.9)$ & $52(26.3)$ & $8(47.1)$ & \\
\hline Other NAC regimens & $3(1.4)$ & $2(1.0)$ & I (5.9) & \\
\hline Neoadjuvant hormonal therapy & $5(2.3)$ & $4(2.0)$ & I (5.9) & \\
\hline
\end{tabular}

(Continued) 
Table I (Continued).

\begin{tabular}{|c|c|c|c|c|}
\hline & Total $n=215$ & No pCR $n=198 ; 92.1 \%$ & pCR $n=17 ; 7.9 \%$ & p value \\
\hline \multicolumn{5}{|l|}{ Breast Surgery } \\
\hline Total mastectomy & $166(77.2)$ & $153(77.3)$ & $13(76.5)$ & 0.940 \\
\hline Breast conserving surgery & $49(22.8)$ & $45(22.7)$ & $4(23.5)$ & \\
\hline \multicolumn{5}{|l|}{ Axillary Surgery } \\
\hline Sentinel node biopsy & $34(15.8)$ & $30(15.2)$ & $4(23.5)$ & 0.319 \\
\hline Axillary dissection & $|8|(84.2)$ & $168(84.8)$ & $13(76.5)$ & \\
\hline \multicolumn{5}{|l|}{ Radiotherapy } \\
\hline No & $19(8.9)$ & $14(7.1)$ & $5(29.4)$ & 0.01 \\
\hline Yes & $195(91.1)$ & $183(92.9)$ & $12(70.6)$ & \\
\hline \multicolumn{5}{|l|}{ Hormonal Therapy } \\
\hline Tamoxifen & $|3|(60.9)$ & $122(61.6)$ & $9(52.9)$ & 0.078 \\
\hline Aromatase inhibitors & $72(33.5)$ & $67(33.8)$ & $5(29.4)$ & \\
\hline Others & $12(5.6)$ & $9(4.5)$ & $3(17.6)$ & \\
\hline
\end{tabular}

Notes: For categorical variables, the $p$ value is obtained from a two-sided Fisher exact test. For continuous variables, the $p$ value is obtained from a two-sided Wilcoxon rank sum test. ${ }^{a} \mathrm{HER} 2$ in situ hybridization was not performed in 44 patients and the patients were categorized into HER2 equivocal group.

Table 2 Magee Equation 2 Score Categories and Outcomes

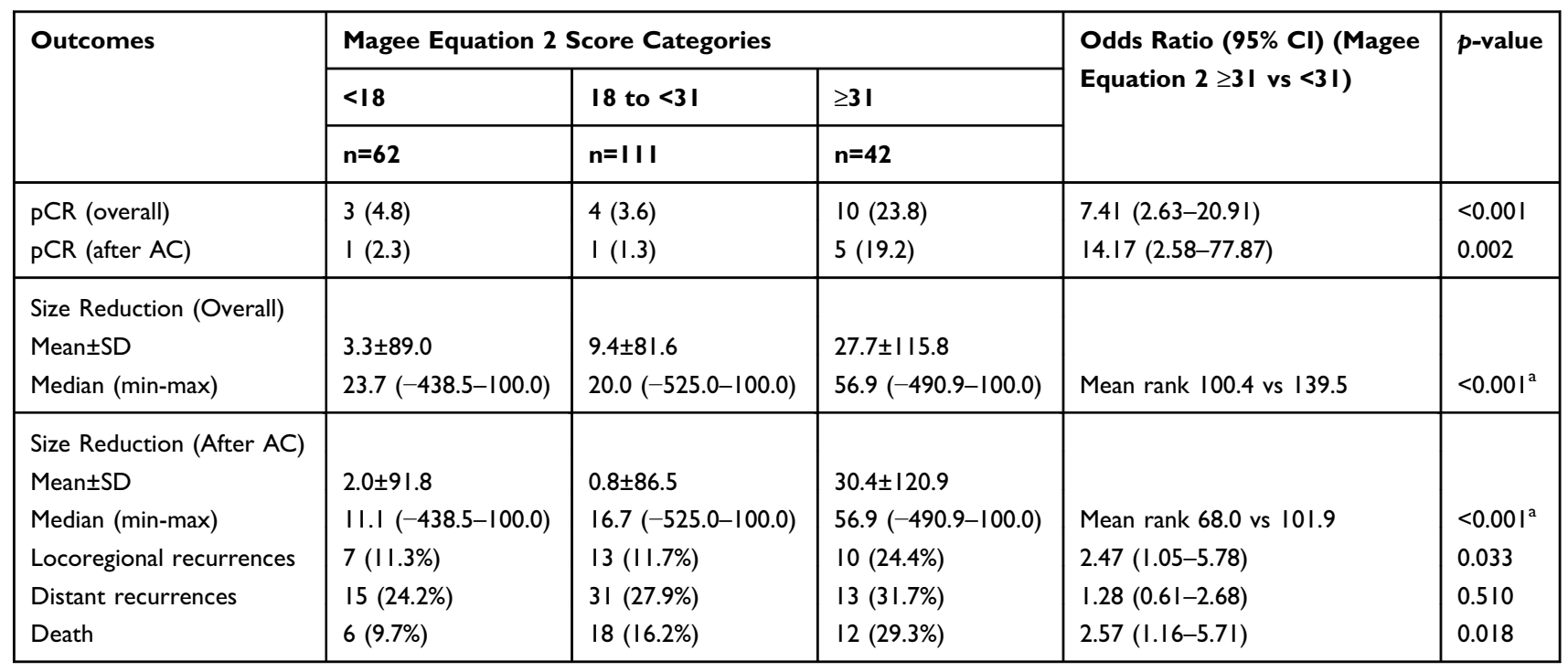

Notes: The $p$ value is obtained from a two-sided Fisher exact test. The confidence interval is obtained from a Wald normal approximation. ${ }^{a}$ Obtained from Mann-Whitney test.

core biopsy might be suitable in this situation. Magee Equation score can accurately estimate the $R S$ and might be used as an alternative predictor for response to chemotherapy. ${ }^{18}$ Magee Equation 3 score was previously reported to be accurately predict the response to NAC in HRpositive, HER2-negative breast cancer. ${ }^{30}$ Due to Ki67 was not available in a significant proportion of the patients in our cohort, Magee Equation 2 that does not require Ki67 results was utilized in the current study. High Magee Equation 2 score was the independent predictor for $\mathrm{pCR}$ in HR-positive,
HER2-negative breast cancer. In contrast, high Magee Equation 2 score was also the independent predictor of lower DFS and OS. The patients with high score had unfavorable clinicopathological parameters and aggressive tumor biology. However, the rate of pCR is also higher in the tumor that had more aggressive biology when compared to the tumor that had favorable biology (eg Luminal breast cancer). Subgroup analysis of the patients with high score showed that among 37 patients with high score, approximately one-fourth of the patients (24.3\%) had pCR. 
Table 3 Multivariate Analysis of Predictive Factors for pCR

\begin{tabular}{|l|l|l|l|l|l|l|l|}
\hline Parameters & B & SE & Wald & Df & p-value & Exp (B) & 95\% CI \\
\hline Age & 0.008 & 0.028 & 0.074 & I & 0.786 & 1.008 & $0.954-1.064$ \\
\hline N0 (ref) & & & 0.330 & 3 & 0.954 & & \\
NI & -0.088 & 0.763 & 0.013 & 1 & 0.908 & 0.915 & $0.205-4.084$ \\
N2 & -0.319 & 0.933 & 0.117 & 1 & 0.733 & 0.727 & $0.117-9.608$ \\
N3 & 0.267 & 1.018 & 0.069 & 1 & 0.793 & 1.305 & $0.177-9.608$ \\
\hline AC-based regimen (ref) & & & 4.704 & 2 & 0.095 & & \\
AC-T regimen & 1.085 & 0.586 & 3.436 & 1 & 0.064 & 2.960 & $0.940-9.327$ \\
Neoadjuvant hormonal therapy & 2.030 & 1.419 & 2.046 & 1 & 0.153 & 7.611 & $0.472-122.779$ \\
\hline Low Magee score (ref) & & & 12.530 & 2 & 0.002 & & \\
Intermediate Magee score & -0.107 & 0.836 & 0.016 & 1 & 0.898 & 0.898 & $0.175-4.621$ \\
High Magee score & 1.987 & 0.785 & 6.402 & 1 & 0.011 & 7.291 & $1.565-33.973$ \\
\hline
\end{tabular}

Lower percentage of death was occurred in the patients with pCR when compared with the patients without pCR $(11.8 \%$ vs $17.2 \%$, respectively). pCR was not a significant predictor for DFS and OS. This finding might be due to a small number of patients with pCR. Furthermore, pooled analysis of more than 11,000 patients among 12 studies suggested that achievement of pCR was associated with better OS in nonluminal (HER2-positive or triple-negative), and grade

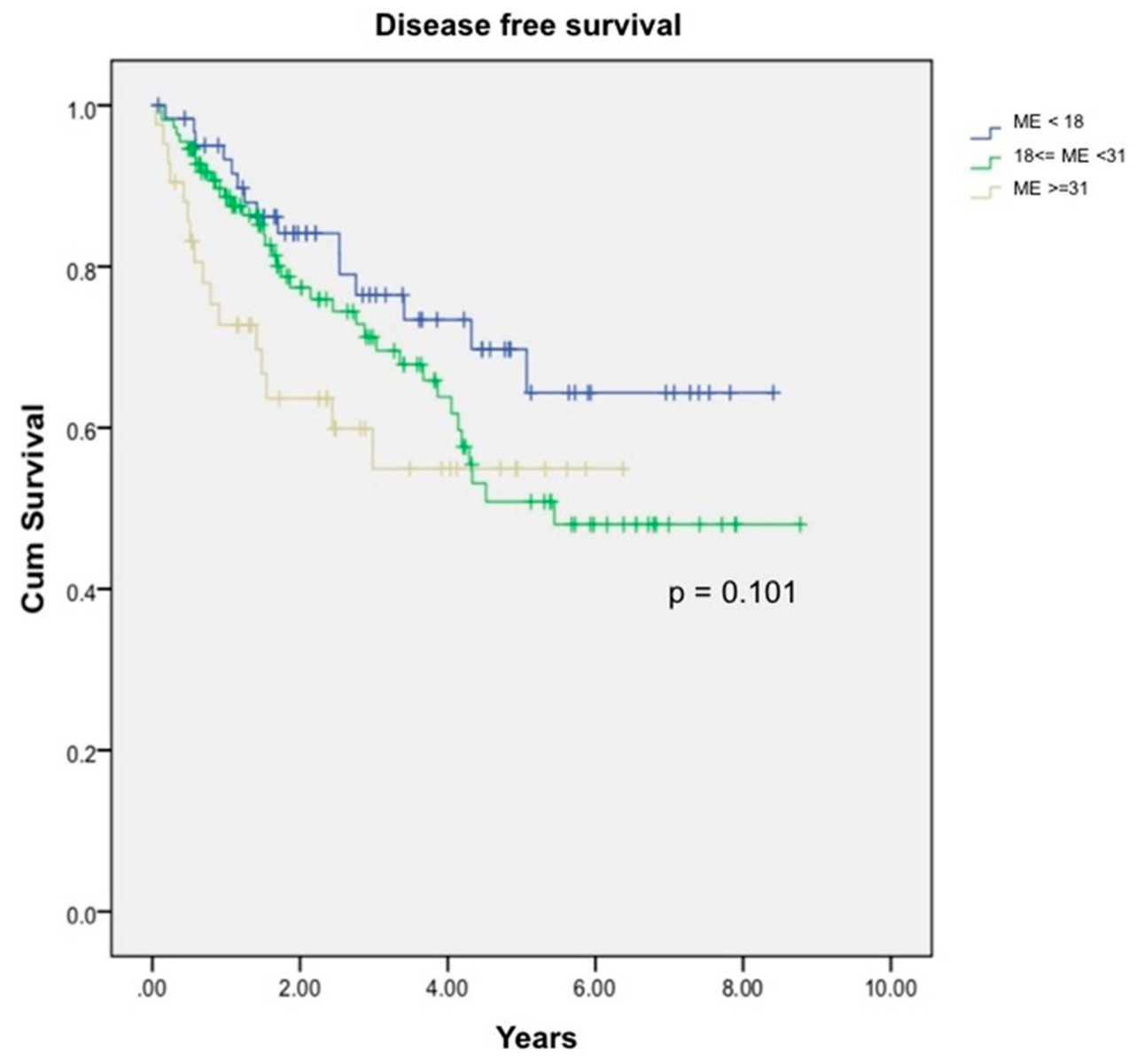

Figure I DFS by Magee Equation 2 score category. ME: Magee Equation 2 score.

Abbreviation: ME, Magee Equation 2 score. 


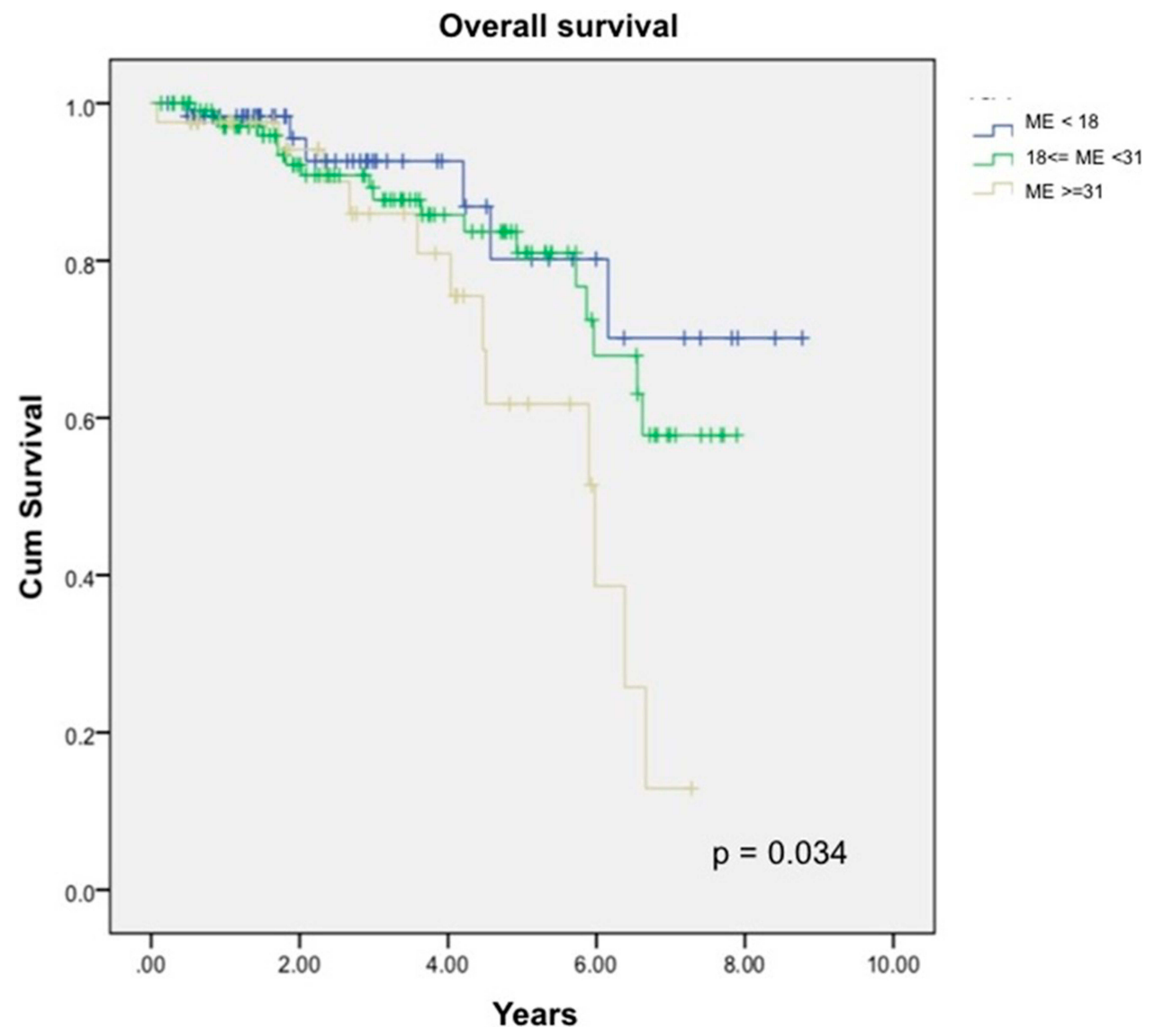

Figure 2 OS by Magee Equation 2 score category.

Abbreviation: ME, Magee Equation 2 score.

3 luminal, HER2-negative breast cancer. In contrast, pCR failed to demonstrate this association in grade 1/2 HRpositive, HER2-negative breast cancer ${ }^{14}$ which accounted for the major proportion of the patients in this current study.
High Magee Equation 2 score was associated with tumor size reduction after NAC. This finding might suggest the application of Magee Equation for prediction of NAC in HRpositive, HER2-negative breast cancer patients who will

Table 4 Multivariate Analysis of Independent Prognostic Factors for DFS

\begin{tabular}{|c|c|c|c|c|c|c|c|}
\hline Parameters & B & SE & Wald & Df & p-value & $\operatorname{Exp}(B)$ & $95 \% \mathrm{Cl}$ \\
\hline Age & -0.000 & 0.014 & 0.001 & I & 0.975 & 1.000 & $0.973-1.027$ \\
\hline No (ref) & & & 12.637 & 3 & 0.005 & & \\
\hline NI & 0.317 & 0.362 & 0.766 & 1 & 0.382 & 1.373 & $0.675-2.792$ \\
\hline N2 & 0.880 & 0.398 & 4.892 & I & 0.027 & 2.410 & I. $105-5.254$ \\
\hline N3 & 1.515 & 0.485 & 9.760 & I & 0.002 & 4.548 & $1.758-11.762$ \\
\hline AC-based regimen (ref) & & & 0.604 & 2 & 0.740 & & \\
\hline AC-T regimen & 0.016 & 0.810 & 0.000 & I & 0.984 & 1.016 & $0.208-4.968$ \\
\hline Neoadjuvant hormonal therapy & 0.247 & 0.828 & 0.089 & I & 0.766 & 1.280 & $0.253-6.483$ \\
\hline Low Magee score (ref) & & & 6.427 & 2 & 0.040 & & \\
\hline Intermediate Magee score & 0.299 & 0.322 & 0.863 & I & 0.353 & 1.349 & $0.717-2.537$ \\
\hline High Magee score & 0.970 & 0.395 & 6.043 & I & 0.014 & 2.639 & $|.217-5.72|$ \\
\hline $\mathrm{PCR}$ & -0.870 & 0.640 & 1.848 & I & 0.174 & 0.419 & $0.119-1.469$ \\
\hline
\end{tabular}


Table 5 Multivariate Analysis of Independent Prognostic Factors for OS

\begin{tabular}{|c|c|c|c|c|c|c|c|}
\hline Parameters & B & SE & Wald & df & p-value & $\operatorname{Exp}(B)$ & $95 \% \mathrm{Cl}$ \\
\hline Age & -0.003 & 0.019 & 0.019 & I & 0.891 & 0.997 & $0.962-1.035$ \\
\hline No (ref) & & & 14.086 & 3 & 0.003 & & \\
\hline NI & 0.371 & 0.531 & 0.490 & I & 0.484 & 1.450 & $0.513-4.101$ \\
\hline N2 & 0.950 & 0.578 & 2.702 & I & 0.100 & 2.587 & $0.833-8.033$ \\
\hline N3 & 2.036 & 0.616 & 10.911 & I & 0.001 & 7.657 & $2.288-25.625$ \\
\hline AC-based regimen (ref) & & & 1.244 & 2 & 0.537 & & \\
\hline AC-T regimen & 0.089 & 1.145 & 0.006 & I & 0.938 & 1.093 & $0.116-10.319$ \\
\hline Neoadjuvant hormonal therapy & $-0.44 I$ & 1.198 & 0.136 & I & 0.713 & 0.643 & $0.062-6.729$ \\
\hline Low Magee score (ref) & & & 8.783 & 2 & 0.012 & & \\
\hline Intermediate Magee score & 0.450 & 0.505 & 0.794 & I & 0.373 & 1.568 & $0.583-4.216$ \\
\hline High Magee score & 1.475 & 0.553 & 7.108 & I & 0.008 & 4.371 & $1.478-12.925$ \\
\hline PCR & -0.794 & 0.798 & 0.990 & I & 0.320 & 0.452 & $0.095-2.159$ \\
\hline
\end{tabular}

receive NAC with the aim of downsizing for conversion from total mastectomy to breast conserving surgery. However, this was not the objective of this study and further study is required to address this issue.

Our study had some limitations as follows. Due to a retrospective study, selection bias might be occurred. Majority of the patients had locally advanced breast cancer and might result in lower $\mathrm{pCR}$ rate. The follow up time was relatively short as the HR-positive breast cancers tend to recur later.

\section{Conclusion}

Our findings demonstrate an association between high Magee Equation 2 scores and pCR after NAC. The Magee Equation 2 might be used for predicting pCR and clinical outcomes in the patients who will receive NAC especially in countries with limited resource for high-cost gene expression assays.

\section{Acknowledgments}

The authors gratefully acknowledge the patients who generously participated in this study. We are grateful to Mr. Suthipol Udompunturak (Faculty of Medicine, Siriraj Hospital) for helping with statistical analysis and appreciated the help from Miss Surat Phumphuang (Department of Surgery, Siriraj Hospital) for coordination of research work and data collection.

\section{Disclosure}

The authors report no conflicts of interest in this work.
The abstract of this paper was presented at the 16th St. Gallen International Breast Cancer Conference as an abstract presentation with interim findings. The poster's abstract was published in "Poster Abstracts" in The Breast: https://doi.org/10.1016/S0960-9776(19)30270-X.

\section{References}

1. Torre LA, Siegel RL, Ward EM, Jemal A. Global cancer incidence and mortality rates and trends-an update. Cancer Epidemiol Biomarkers Prev. 2016;25(1):16-27. doi:10.1158/1055-9965.EPI-15-0578

2. Calhoun BC, Collins LC. Predictive markers in breast cancer: an update on ER and HER2 testing and reporting. Semin Diagn Pathol. 2015;32(5):362-369. doi:10.1053/j.semdp.2015.02.011

3. Wang WJ, Lei YY, Mei JH, Wang CL. Recent progress in HER2 associated breast cancer. Asian Pac J Cancer Prev. 2015;16 (7):2591-2600. doi:10.7314/APJCP.2015.16.7.2591

4. Banin Hirata BK, Oda JM, Losi Guembarovski R, Ariza CB, de Oliveira CE, Watanabe MA. Molecular markers for breast cancer: prediction on tumor behavior. Dis Markers. 2014;2014:513158. doi: $10.1155 / 2014 / 513158$

5. Sheri A, Dowsett M. Developments in Ki67 and other biomarkers for treatment decision making in breast cancer. Ann Oncol. 2012;23(Suppl 10):x219-x227. doi:10.1093/annonc/mds307

6. Sonnenblick A, Francis PA, Azim HA Jr., et al. Final 10-year results of the Breast International Group 2-98 Phase III trial and the role of Ki67 in predicting benefit of adjuvant docetaxel in patients with oestrogen receptor-positive breast cancer. Eur J Cancer. 2015;51(12):1481-1489. doi:10.1016/j.ejca.2015.03.018

7. Li CI, Daling JR, Malone KE. Incidence of invasive breast cancer by hormone receptor status from 1992 to 1998. J Clin Oncol. 2003;21 (1):28-34. doi:10.1200/JCO.2003.03.088

8. Early Breast Cancer Trialists' Collaborative Group (EBCTCG). Effects of chemotherapy and hormonal therapy for early breast cancer on recurrence and 15-year survival: an overview of the randomised trials. Lancet (London, England). 2005;365(9472):1687-1717. doi:10.1016/S0140-6736(05)66544-0

9. Davies C, Godwin J, Gray R, et al. Relevance of breast cancer hormone receptors and other factors to the efficacy of adjuvant tamoxifen: patient-level meta-analysis of randomised trials. Lancet (London, England). 2011;378(9793):771-784. 
10. Sparano JA, Paik S. Development of the 21-gene assay and its application in clinical practice and clinical trials. J Clin Oncol. 2008;26(5):721-728. doi:10.1200/JCO.2007.15.1068

11. Fisher B, Jeong JH, Dignam J, et al. Findings from recent National Surgical Adjuvant Breast and Bowel Project adjuvant studies in stage I breast cancer. J Natl Cancer Inst Monogr. 2001;2001(30):62-66. doi:10.1093/oxfordjournals.jncimonographs.a003463

12. Specht J, Gralow JR. Neoadjuvant chemotherapy for locally advanced breast cancer. Semin Radiat Oncol. 2009;19(4):222-228. doi:10.1016/j. semradonc.2009.05.001

13. Asselain B, Barlow W, Bartlett J; Early Breast Cancer Trialists' Collaborative G. Long-term outcomes for neoadjuvant versus adjuvant chemotherapy in early breast cancer: meta-analysis of individual patient data from ten randomised trials. Lancet Oncol. 2018;19 (1):27-39. doi:10.1016/S1470-2045(17)30777-5

14. Cortazar P, Zhang L, Untch M, et al. Pathological complete response and long-term clinical benefit in breast cancer: the CTNeoBC pooled analysis. Lancet. 2014;384(9938):164-172. doi:10.1016/S01406736(13)62422-8

15. Paik S, Shak S, Tang G, et al. A multigene assay to predict recurrence of tamoxifen-treated, node-negative breast cancer. $N$ Engl J Med. 2004;351(27):2817-2826. doi:10.1056/NEJMoa041588

16. Gianni L, Zambetti M, Clark K, et al. Gene expression profiles in paraffin-embedded core biopsy tissue predict response to chemotherapy in women with locally advanced breast cancer. J Clin Oncol. 2005;23(29):7265-7277. doi:10.1200/JCO.2005.02.0818

17. Flanagan MB, Dabbs DJ, Brufsky AM, Beriwal S, Bhargava R. Histopathologic variables predict Oncotype DX recurrence score. Mod Pathol. 2008;21(10):1255-1261. doi:10.1038/modpathol.2008.54

18. Klein ME, Dabbs DJ, Shuai Y, et al. Prediction of the Oncotype DX recurrence score: use of pathology-generated equations derived by linear regression analysis. Mod Pathol. 2013;26(5):658-664. doi:10.1038/modpathol.2013.36

19. Turner BM, Skinner KA, Tang P, et al. Use of modified Magee equations and histologic criteria to predict the Oncotype DX recurrence score. Mod Pathol. 2015;28(7):921-931. doi:10.1038/ modpathol.2015.50

20. Harowicz MR, Robinson TJ, Dinan MA, et al. Algorithms for prediction of the Oncotype DX recurrence score using clinicopathologic data: a review and comparison using an independent dataset. Breast Cancer Res Treat. 2017;162(1):1-10. doi:10.1007/s10549-016-4093-4
21. Mazouni C, Peintinger F, Wan-Kau S, et al. Residual ductal carcinoma in situ in patients with complete eradication of invasive breast cancer after neoadjuvant chemotherapy does not adversely affect patient outcome. J Clin Oncol. 2007;25(19):2650-2655. doi:10.1200/JCO.2006.08.2271

22. McCarty KS Jr., Miller LS, Cox EB, Konrath J, McCarty KS. Sr. Estrogen receptor analyses. Correlation of biochemical and immunohistochemical methods using monoclonal antireceptor antibodies. Arch Pathol Lab Med. 1985;109(8):716-721.

23. Spring LM, Fell G, Arfe A. et al. Pathological complete response after neoadjuvant chemotherapy and impact on breast cancer recurrence and survival: a comprehensive meta-analysis. Clin Cancer Res;2020. clincanres.3492.2019. doi:10.1158/1078-0432.CCR-19-3492

24. Choi HJ, Ryu JM, Kim I, et al. Nomogram for accurate prediction of breast and axillary pathologic response after neoadjuvant chemotherapy in node positive patients with breast cancer. Ann Surg Treat Res. 2019;96(4):169-176. doi:10.4174/astr.2019.96.4.169

25. Kim HS, Yoo TK, Park WC, Chae BJ. Potential benefits of neoadjuvant chemotherapy in clinically node-positive luminal subtype(-) breast cancer. J Breast Cancer. 2019;22(3):412-424. doi:10.4048/ jbc.2019.22.e35

26. Chatterjee D, Bal A, Das A, Singh G. Proliferation rate and breast cancer subtype, but not ALDH1 expression, predict pathological response to neoadjuvant chemotherapy in locally advanced breast cancer. Virchows Arch. 2015;467(3):303-310. doi:10.1007/s00428-015-1794-8

27. Diaz-Casas SE, Castilla-Tarra JA, Pena-Torres E, et al. Pathological response to neoadjuvant chemotherapy and the molecular classification of locally advanced breast cancer in a Latin American Cohort. Oncologist. 2019;24(12):e1360-e1370. doi:10.1634/theoncologist.2019-0300

28. Rastogi P, Anderson SJ, Bear HD, et al. Preoperative chemotherapy: updates of National Surgical Adjuvant Breast and Bowel Project Protocols B-18 and B-27. J Clin Oncol. 2008;26(5):778-785. doi:10.1200/JCO.2007.15.0235

29. Yardley DA, Peacock NW, Shastry M, et al. A Phase II trial of ixabepilone and cyclophosphamide as neoadjuvant therapy for patients with HER2-negative breast cancer: correlation of pathologic complete response with the 21-gene recurrence score. Breast Cancer Res Treat. 2015;154(2):299-308. doi:10.1007/s10549-015-3613-y

30. Farrugia DJ, Landmann A, Zhu L, et al. Magee Equation 3 predicts pathologic response to neoadjuvant systemic chemotherapy in estrogen receptor positive, HER2 negative/equivocal breast tumors. Mod Pathol. 2017;30(8):1078-1085. doi:10.1038/modpathol.2017.41

\section{Publish your work in this journal}

Cancer Management and Research is an international, peer-reviewed open access journal focusing on cancer research and the optimal use of preventative and integrated treatment interventions to achieve improved outcomes, enhanced survival and quality of life for the cancer patient.
The manuscript management system is completely online and includes a very quick and fair peer-review system, which is all easy to use. Visit http://www.dovepress.com/testimonials.php to read real quotes from published authors. 